\title{
BILATERAL BREAST SARCOMA IN WOMEN WITH MACROMASTIA
}

\author{
Igor Galaychuk \\ Department of Oncology and Radiology \\ Ternopil State Medical University \\ 8 R. Kupchynsky Str., Ternopil, Ukraine, 46023 \\ Larysa Nitefor \\ Department of Oncology and Radiology \\ Ternopil State Medical University \\ 8 R. Kupchynsky Str., Ternopil, Ukraine, 46023 \\ Irina Perepyolkina \\ CSD Health Care Medical Lab \\ 45 Vasylkivska Str., Kyiv, Ukraine, 03022
}

\begin{abstract}
Bilateral breast sarcomas are rare malignancies of unknown etiology. Most common histological subtypes of breast sarcoma are angiosarcoma, fibrosarcoma and malignant fibrous histiocytoma. Phyllodes tumors have a predisposition to transform into sarcoma.

The present clinical case describes bilateral breast fibrosarcomas in a 39 years-old female with macromastia. This peculiarity gave possibilities to do wide local excision of both tumors within $3.0 \mathrm{~cm}$ resection margins and preserve both breasts. Histological and immunohistochemical (ERG, CK-AE1/AE3) examination confirmed fibrosarcomas in both breast (in right - stage IIB, in left stage IIA). Patient carried on postoperative treatment with radiation therapy (36.1 Gy for both breasts) and four cycles of adjuvant chemotherapy (doxorubicin, cisplatin, cyclophosphamide).

For the first year patient had clinical and ultrasound examination every three months, then every 6 months. CT scan of chest and abdomen was every year. In seven post-op years there was no evidence of disease recurrence.

Thus, macromastia gives an exceptional chance to carry out organ-sparing radical surgery in women with advanced breast malignancies.
\end{abstract}

Keywords: breast sarcomas, breast conservative surgery, macromastia.

\section{Introduction}

Breast sarcomas consist less than $1.0 \%$ of all breast malignancies and less than $5.0 \%$ all soft tissue sarcomas. The most common histological subtypes of breast sarcoma were angiosarcoma, fibrosarcoma and malignant fibrous histiocytoma $[1,2]$.

There are only several publications concerning bilateral breast sarcomas (BBS) as primary diseases, radiation induced and metastatic - all with a fatal outcome [3-7].

Therefore, BBS are high aggressive malignant tumors, and nowadays a practical recommendation about theirs surgery and adjuvant treatment absent. That is why every clinical case is appreciated.

\section{Aim of research}

To describe the successful clinical case of BBS in patient with macromastia.

\section{Materials and methods}

In 2010, 39 years-old female was diagnosed with tumors in both breasts. Palpation, sonography and mammography revealed in the right breast tumor with dimension $55 \times 50 \mathrm{~mm}$, in left one $40 \times 30 \mathrm{~mm}$. In the right breast the tumor located in the middle of lower quadrants; in the left breast the tumor was close to submammary fold and fixed to underlain soft tissues. Cytological exam- 
ination after fine needle aspiration biopsy found malignant cells in both tumors. Axillaries lymph nodes were not enlarged according to ultrasound exam.

The peculiarities of this case consist in large size of breasts (E-cap, macromastia) that cause late diagnosis. In age $31 \mathrm{yr}$. woman underwent surgery - lumpectomy of right breast due to phyllodes fibroadenoma which was histologically confirmed.

Given the large correlation between the volume of breasts (macromastia) and the volume of both tumors, as well as a considerable distance $(27-30 \mathrm{~cm})$ from the tumor to the regional lymphatic nodes and the absence of lymph node involvement, the organ sparing operations were planned.

Preoperative diagnosis: bilateral breast sarcomas?

Surgery: wide local excision of the tumor in right breast within $3.0 \mathrm{~cm}$ margins, and "en block" excision of the left breast tumor with resection of submammary fold, soft chest tissue and part of muscle pectoralis major.

Visual exam of surgical specimens confirmed the sarcomatoid origin of both tumors (Fig. 1). Healing of postoperative wounds was without any complications.

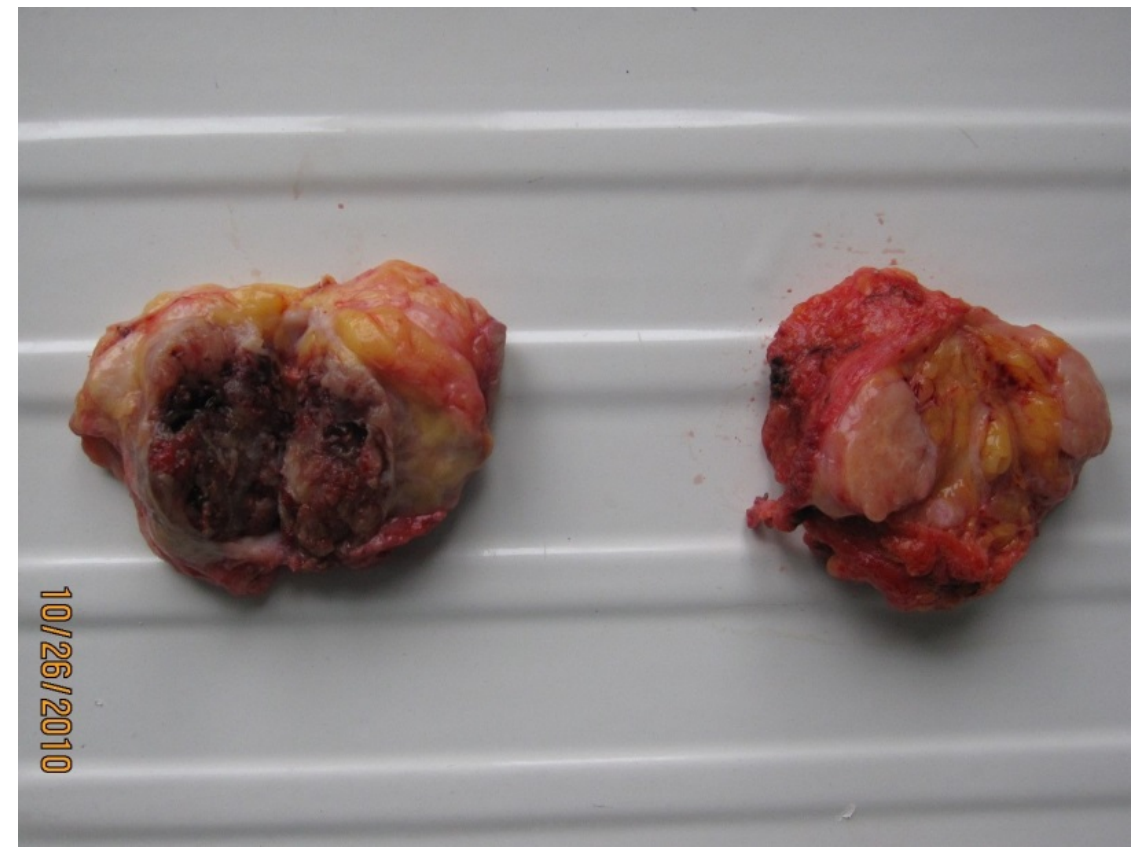

Fig. 1. Surgical specimens of both breast sarcomas - right and left (2010)

Pathohistological examination: fibrosarcoma of the right breast, G3; fibrosarcoma of the left breast with myxomatosis, G3 (Fig. 2, 3). The edges of resection were "clear" in both surgical specimens.

Post-op diagnosis (TNM-6):

1) Fibrosarcoma of the right breast, pT2aN0M0G3, stage IIB;

2) Fibrosarcoma of the left breast, pT1bN0M0G3, IIA stage.

The immunohistochemistry (IHC) specification was done for the differential diagnosis between epithelial and non-epithelial malignancies. For these purpose the IHC with ERG and multi-cytoceratin (clones AE1/AE3) were used.

Erythroblast transformation specific related gene $(E R G)$ is a sensitive marker of endothelial differentiation and is expressed in vascular tumours, including angiosarcomas. In our case ERG marker was negative in tumours cells (Fig. 3, a), it means that tumours not belong to angisarcoma.

Cytokeratins (CK) are important markers for the IHC classification of undifferentiated tumors. Non-epithelial tumors, as a rule, are cytokeratin-negative. To identify cytokeratins in tumor cells, so-called multi-cytokeratin clones AE1/AE3 - antibodies with a wide specificity for different epithelia are often used. They can be used for the differential diagnosis of cancer with 
the majority of non-epithelial tumors. In our patient the tumor cells were negative cytoceratin AE1/AE3 stains (Fig. 3, b).

Thus, histological structure of tumors and their immunophenotype correspond to fibrosarcoma.

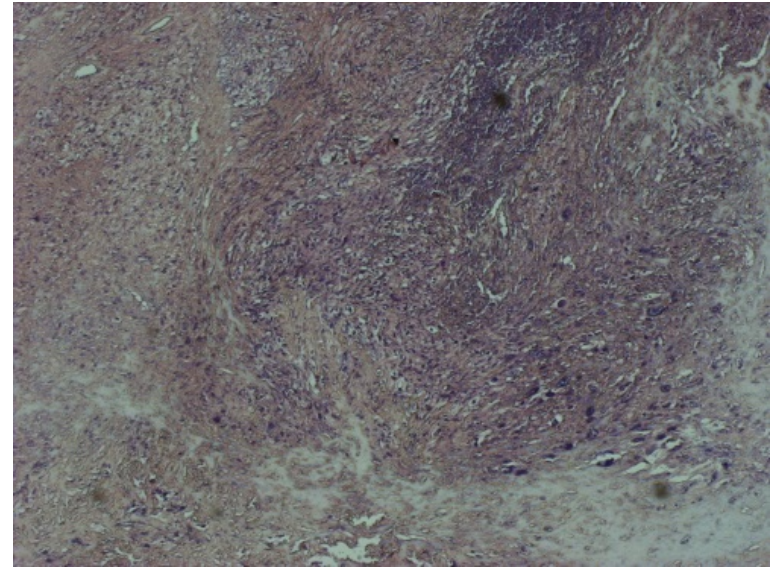

$a$

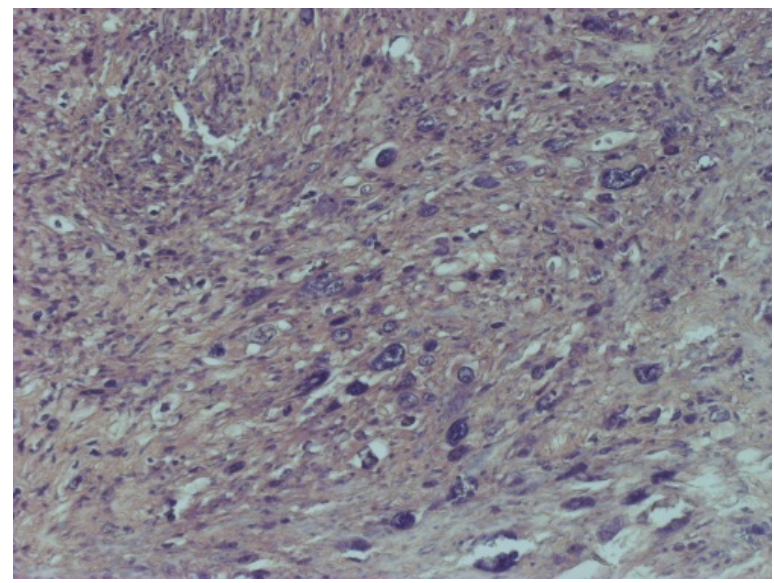

$b$

Fig. 2. Histological microphotographs (H\&E): $a$ - fibrosarcoma of the right breast $(\times 40)$; $b$ - fibrosarcoma of the left breast $(\times 100)$

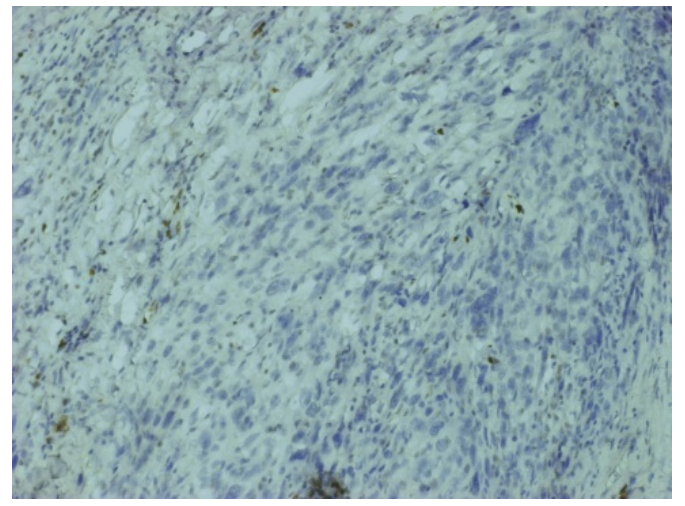

$a$

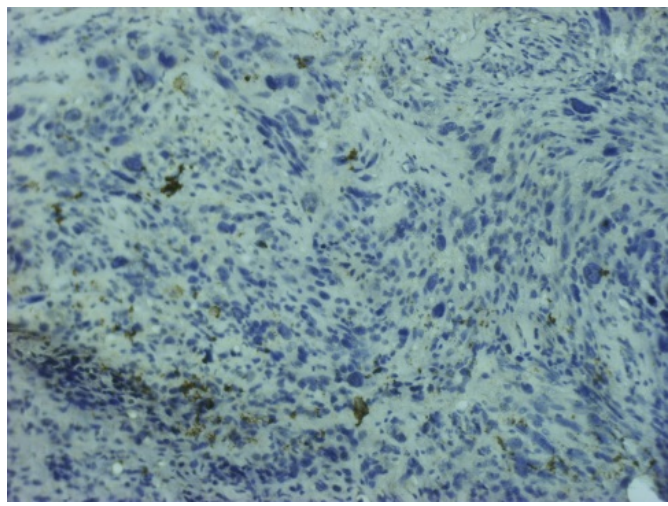

$b$

Fig. 3. IHC ( $\times 200): a-$ ERG (Dako, clon EP111): tumour cells negative; cells like small lymphocytes and vessels' endothelial cells are positive; ): $b-\mathrm{CK}$ : tumor cells are negative for multi-cytoceratin AE1/AE3 stain 
Adjuvant treatment. Patient had radiation treatment and chemotherapy in post-op period. External beam radiation gamma-therapy (Co-60) was performed for both breasts with doses in 36.1 Gy. Four cycles of adjuvant chemotherapy (doxorubicin, cisplatin, cyclophosphamide) were realized.

\section{Results and discussion}

Follow-up. For the first year patient had clinical and ultrasound examination of breast and regional lymph nodes every three months, then every 6 months. CT scan of chest and abdomen was every year. In five (2015; Fig. 4) and seven (2017) post-op years no local recurrences or distant metastases were detected.

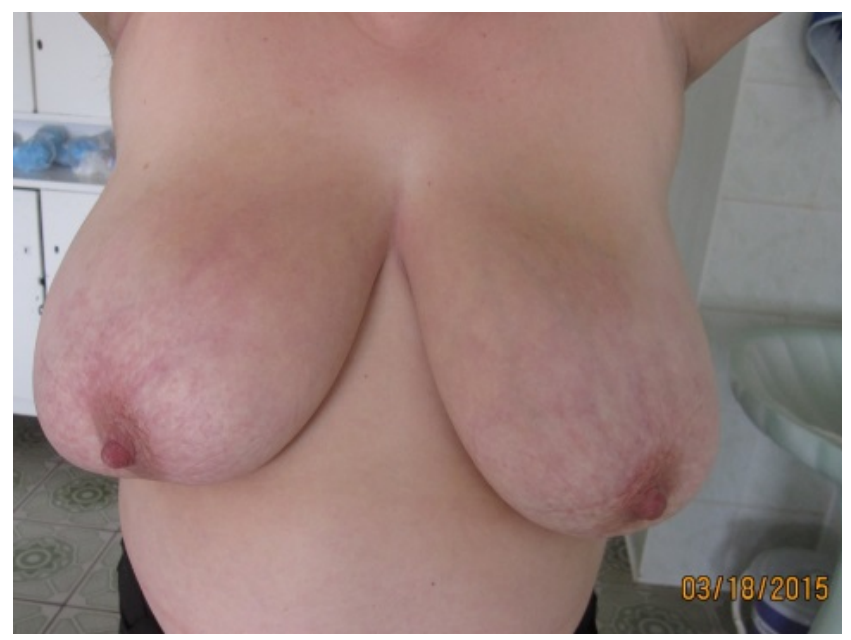

$a$

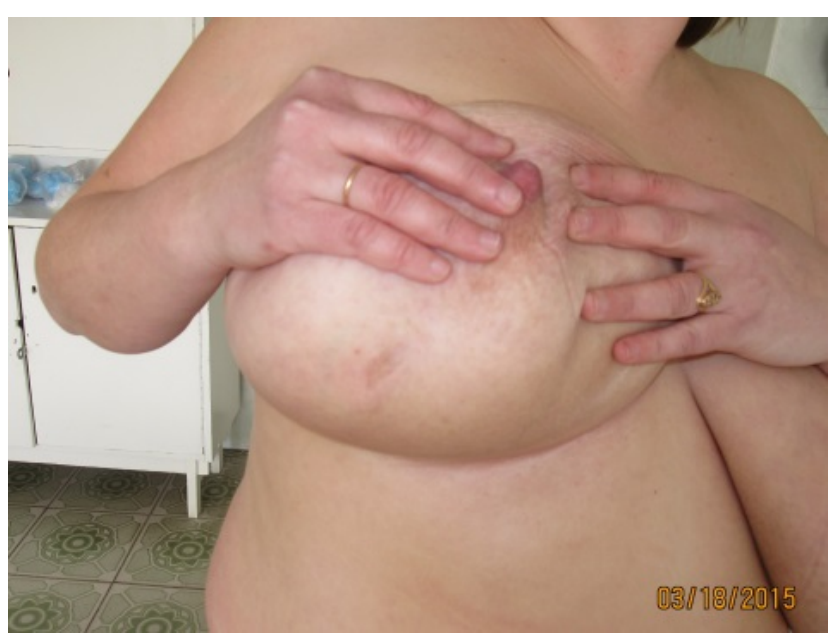

$b$

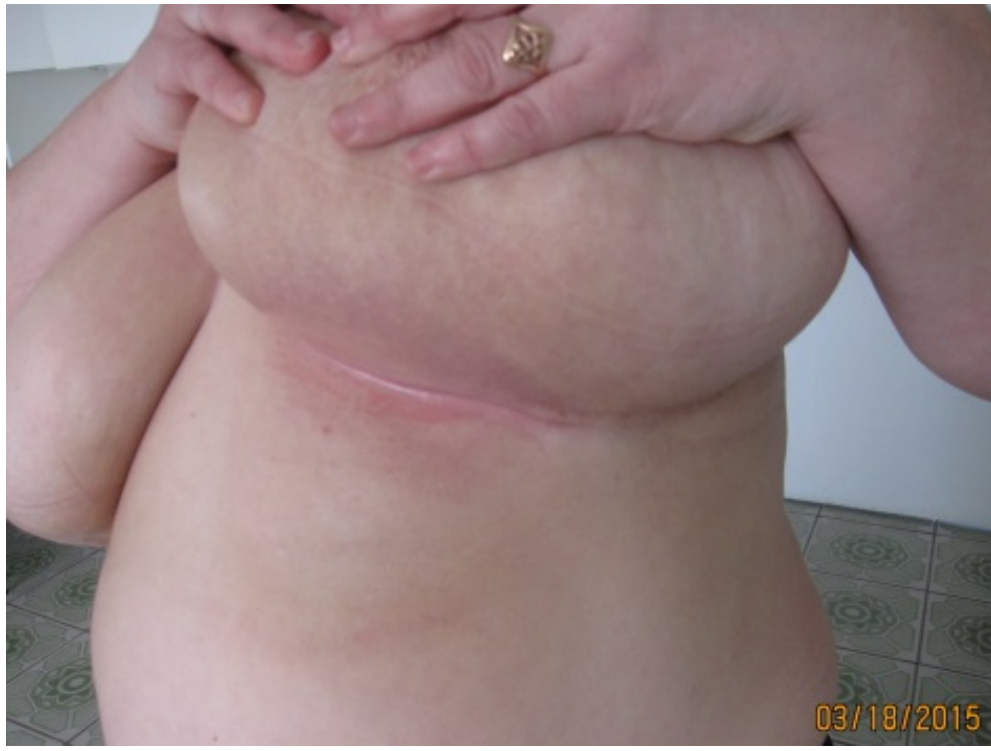

c

Fig. 4. The view of breasts in 5 post-op years: $a$-general view of breasts;

$b$ - surgical scar on the right breast; $c$ - surgical scar on the left submammary fold

Breast sarcomas represent a heterogeneous group of tumors of the mesenchymal origin with an extremely aggressive course. Fibrosarcoma is accounting about $16.0 \%$ of all breast sarcomas. Contralateral breast involvement may be due to either development of a secondary primary tumor or metastatic spread [1, 3-6]. 
The lumpectomy or partial mastectomy is preferred surgery in patients with breast sarcomas. Mastectomy is recommended for large tumors $(>5.0 \mathrm{~cm})$ or when there are positive resection margins after local excision [1-4, 8]. In our clinical case, radical surgeries were performed with preservation of the breasts and without axillaries dissections.

Radiotherapy is recommended in the case of high-grade tumor and after conservative breast surgery or in the case of positive surgical margins [1, 3, 4]. As to chemotherapy it is used in case of high grade or metastatic sarcomas for 4-6 cycles. Nowadays the cytostatics are combined with targeted therapy $[2,6]$. Considering our clinical case, surgery, radiation therapy and chemotherapy were performed in the same way as for soft tissue sarcoma.

It is very important to differentiate the metaplastic carcinoma from soft tissue sarcomas. This can be done both by histological and IHC exam with cytokeratin positivity of the metaplastic cells. Absence of any epithelial component and negative staining of a panel of immunomarkers helped the diagnosis of fibrosarcoma [2, 3].

In the case of phyllodes tumors, wide excision with adequate margins is essential to the prevention of local recurrence and to provide an accurate diagnosis as to whether it is benign, borderline or malignant (mitotic activity, stromal cell hyperplasia and atypia). It is essential that borderline and malignant phyllodes tumors may metastasize [9-12].

In summary, the therapeutic approach for breast sarcomas should be carried out according to the principles of treatment of soft tissue sarcoma, taking into account the anatomical features of the breast.

\section{Conclusions}

Our experience show that in the case of bilateral breast fibrosarcomas in women with macromastia the aggressive treatment approach should be done - radical wide local excision of tumors with postoperative radiation and chemo-therapy.

Macromastia gives an exceptional chance to carry out organ-sparing radical surgery in woman with advanced breast malignancies.

\section{References}

[1] Adem, C., Reynolds, C., Ingle, J. N., Nascimento, A. G. (2004). Primary breast sarcoma: clinicopathologic series from the Mayo Clinic and review of the literature. British Journal of Cancer, 91, 237-241. doi: 10.1038/sj.bjc.6601920

[2] Lim, S. Z., Ong, K. W., Tan, B. K. T., Selvarajan, S., Tan, P. H. (2016). Sarcoma of the breast: an update on a rare entity. Journal of Clinical Pathology, 69 (5), 373-381. doi: 10.1136/jclinpath-2015203545

[3] Pai, M., Upadhyaya, K., Naik, R., Malhotra, S. (2008). Bilateral angiosarcoma breast diagnosed by fine needle aspiration cytology. Indian Journal of Pathology and Microbiology, 51 (3), 421. doi: 10.4103/0377-4929.42549

[4] De Bree, E., van Coevorden, F., Peterse, J. L., Russell, N. S., Rutgers, E. J. T. (2002). Bilateral angiosarcoma of the breast after conservative treatment of bilateral invasive carcinoma: genetic predisposition? European Journal of Surgical Oncology (EJSO), 28 (4), 392-395. doi: 10.1053/ejso.2001.1249

[5] Zhou, S.-A., Wei, H., Ding, K. (2009). A Rare Case of Metachronous Bilateral Angiosarcoma of the Breast. Breast Care, 4 (6), 405-407. doi: 10.1159/000261506

[6] Astudillo, L., Lacroix-Triki, M., Ferron, G., Rolland, F., Maisongrosse, V., Chevreau, C. (2005). Bilateral Breast Metastases From Ewing Sarcoma of the Femur. American Journal of Clinical Oncology, 28 (1), 102-103. doi: 10.1097/01.coc.0000149741.46332.17

[7] Kwatra, K. S., Prabhakar, B. R., Arora, Y. (2004). Bilateral granulocytic sarcoma (chloroma) of the breast in CML in blast crisis: a case report. Indian Journal of Pathology \& Microbiology, 47 (1), 66-68.

[8] Grenier, J., Delbaldo, C., Zelek, L., Piedbois, P. (2010). Phyllodes Tumors and Breast Sarcomas: A Review. Bull Cancer, 97 (10), 1197-1207. 
[9] Warrier, S., Hwang, S. Y., Gibbings, K., Carmalt, H., O’Toole, S. (2015). Phyllodes tumour with heterologous sarcomatous differentiation: Case series with literature review. International Journal of Surgery Case Reports, 11, 91-94. doi: 10.1016/j.ijscr.2015.02.009

[10] Jang, J. H., Choi, M.-Y., Lee, S. K., Kim, S., Kim, J., Lee, J. et. al. (2012). Clinicopathologic Risk Factors for the Local Recurrence of Phyllodes Tumors of the Breast. Annals of Surgical Oncology, 19 (8), 2612-2617. doi: 10.1245/s10434-012-2307-5

[11] Shaaban, M., Barthelmes, L. (2017). Benign phyllodes tumours of the breast: (Over) treatment of margins - A literature review. European Journal of Surgical Oncology (EJSO), 43 (7), 1186-1190. doi: 10.1016/j.ejso.2016.10.019

[12] Zhou, Z.-R., Wang, C.-C., Yang, Z.-Z., Yu, X.-L., Guo, X.-M. (2016). Phyllodes tumors of the breast: diagnosis, treatment and prognostic factors related to recurrence. Journal of Thoracic Disease, 8 (11), 3361-3368. doi: 10.21037/jtd.2016.11.03 